\title{
IMPORTANCE OF STEP IN THE INTEGRATION OF MANUFACTURING ACTIVITIES
}

\author{
Ashok.G \\ BKIT, Bhalki, Karnataka State, India
}

\begin{abstract}
In achieving the CIM, Computer manufacturing and computer aided process planning seamlessly is one of the main problem. For generating product design CAD systems have more tendency keenly than on readily and visibly facilitating the development of a product. At present there is a noticeable require in the souk for computer aided design and manufacturing systems that effortlessly put together and assist design and manufacturing actions, this allows for design changes on the fly, exchange of number element information and competently accepting tool using extremely different machines and systems. Transfer of data between Computer aided design, computer aided manufacturing systems and computer aided process planning is the vital concern in the integration. In the integration how the part data exchange standard is used, we discussed in this paper, with this in the development of STEEP $C A D / C A P P / C A M \& C N C$ integrated system what is the role of standard for exchange of product data has been studied.
\end{abstract}

Keywords: CAD, CAPP, CAM, Step, Product Model Data, Exchange of Data

$* * *$

\section{INTRODUCTION}

With the advancement in technology and improvement in the capability of computer systems, for rising production effectiveness and manufacturing flexibility and to support manufacturing lots of computers aided systems are used in the industries. Every computer aided system has its individual interior statistics of parts. These manufactured goods data might be different from one to another system in their formats. Production engineering of manufactured goods, Different sections of industries will be busy. In speckled sites different calculating applications are used. The date information regarding product will be exchanged among them. This illustrates the approach to the principles for product replica information exchange Bloor, M. S., 1991]

For of the product life cycle such as design, the CAD, CAPP and CAM system supports a autonomous phase., each system shall work jointly In the computer integrated manufacturing environments to support the life cycle of a product, as a result the production efficiency can be improved to a vast level. Main component of a CIM system are An integrated CAD /CAPP/CAM system. Because of these reasons integration troubles become highlight of systems improvement. The product model information swap above between CAD, CAPP and CAM systems is the respond to the system integration.

A standard for product model data representation and exchange In 1983, ISO started a project with regard this mater. The assignment is performed by ISO TC184/SC4, and this named as STEP (Standard for the Exchanging of Product Model data). to represent the total product data which totally describe the product for all applications over its predictable life sequence, and to afford methods for swap over, giving out, and archiving of product data is assured by STEP. In the integration of CAD/CAPP/CAM systems STEP can take part which are expected to be used in a CIM atmosphere.

\section{STEP GROUND RULES}

STEP is a worldwide set for the computer-interpretable illustration and swap over of product model data. The major plan is to give a neutral system proficient of illumination of product data for the period of the life cycle of a product, free from some meticulous system. The mode in which it describes makes it fit not only for a foundation for applying and giving out product databases and archiving, also neutral file.

The method formed concerning a product during its design, developed, consumption, preservation and dumping is used for several purposes during its life cycle similar to Product Data Management, Product Life Cycle Management, development, examination, etc. The use may engage many computer systems. The information of the product is to be obtainable in a general computer interpretable configuration that is required to keep on absolute and dependable when exchanged in special computer aided systems. STEP is presently to assemble this necessity. Activity or doubling of product data between systems leads to inconsistencies and doses not include importance.

STEP is planned as a sequence of parts, every published independently. The parts drop into one of the following series: The EXPRESS is the language in STEP as a description method for specifies the presentation of product information. Hold up for CAD/CAPP/ CAM \& CNC integration, they 
showed records of STEP have rich contents that can be a sound. The integrated property consists of topology, basics, manufactured goods, geometry prototype, material, acceptance, form aspect, etc. aspects of product details the whole time the life cycle. Numerous function protocols have been summarized. Achievement methods of file swap and appliance programming edge are accessible.

STEP allows the manufacturers to get new, better quality and productivity while reducing costs and time-to-market. The figure below shows the structure of STEP.

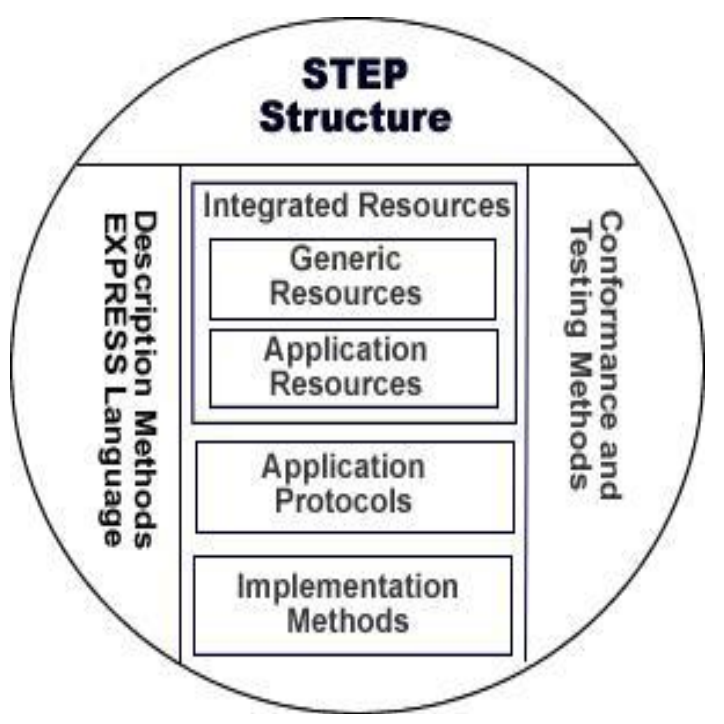

Fig 1 Structure of STEP

\section{ROLE OF STEP IN THE INTEGRATION}

The major problem involved in the system integration is, exchange of product data between CAD, CAPP and CAM and systems seamlessly. There are two conditions for exchange of product data. 1) Common product model to CAD, CAPP and CAM systems. 2) Implementation mechanism for data exchange. Both finds support in STEP.

For a range of segments of propose process planning, replica, and assemble correspondingly, CAPP, CAD, and CAM systems maintain. The product data of every computer system deals with are restricted to a section of the whole product data model. e.g., CAD system will core on geometry shape data. A worldwide product data model is necessary, which every system allocates general definitions. The product data that each system processes can be a part of the global product data model.

The STEP integrated resources are a common illustration of product details right through the life cycle of product. This illustration of product data is unmistakable, absolute, and computer-interpretable. It positively wires the design, method arrangement and construct of a product. The worldwide product data design for CAPP/CAD//CAM included system may be extracted from the integrated possessions, with extra limitations interpreted for definite applications of CAD/CAPP/CAM system. The emblematic data of a motorized product and the interconnected Parts of integrated assets are:

Figure explanation:

Element 42 (figure and topology),

Element 47 (figure tolerances),

Element 48 (structure properties);

\section{Substantial belongings report:}

Element 45 (resources);

\section{Product managerial information narrative:}

Element 41 (Product explanation and maintain), Element 44 (product composition arrangement)

The global product data model shall be the totality of data of a product on design, process planning and manufacture [U.S. Product Data Association, Part 41, 1994]. It consists of several EXPRESS schemas, in which resource constructs such as EXPRESS entities reside.

Two mechanisms used for interchanging the information given by the step execution methods: application encoding interface and clear text encoding of interchange structure. Through the interchange records clear text technique encodes transferring the information. Through the preprocessor system converts its creation replica information into the STEP swap over for the STEP file. With the related postprocessor it takes the information as of the STEP. Depend on the STEP file design and the product facts model of the interchanged information, the processors are created. For the information interchange $\mathrm{cpp} / \mathrm{cam} / \mathrm{cad}$ integrated system, among the following system capp, cad or cam which wants to send the product information can have a preprocessor, other side a system wants to receive the informational shall have a postprocessor. For the self-cycle analysis a system must have mutually the preprocessor ad the postprocessor and use of information interchange

The purpose Programming crossing point of STEP is a regular data right to use edge for encoding. A set of data access functions implements the data access operations on a product data repository. The used product information seen to be designed in EXPRESS word. This method is one of the most effective techniques for system integration. Using the CAD system we can put aside the product structure information in the data storage area via the information saving method and the CAM technique get the shape data via the information getting operation. The creation information storage may be a file system or data base, but this must sustain the services of data access explained in the standard access interface. 


\section{STEP USES IN THE CAD/CAPP/CAM INTEGRATION}

An integrated product information model obtained by the STEP integrated resources entire life cycle of a product. The element of this data replica is worried for a definite appliance area. Uninhibited implementations would outcome if the construction bit was random in such huge standard. To come out from such a problem, STEP gives the application of a set of rules. An application protocol having precise information models for some different application context. Using the STEP, record of entities as in integrated resources, situation in which they are to used and a mapping representing which exacting task they carry out in the application. [Smith, B. M, 1989].

Four main parts on which Application protocols are based:

- prospect and situation of a specific application;

- An application orientation model (ARM) which tells the data necessities of the application properly;

- An application interpreted model (AIM) from using the resource constructs from integrated resources to assure the requirements specified in the ARM;

- Conformance requirements and test purposes for implementations complying with the application protocols [U.S. Product Data Association, Part 203, 1994].

To produce a STEP execution, an application protocol is joined with a particular implementation form.

For CAD/CAPP/CAM integration, AP,s are required for several application contexts. These applications may be categorized into two classes. One is on a certain segment of the life cycle of a product, such as design, process planning, or manufacture. The AP's for applications in this class support each system of CAD, CAPP and CAM independently. The other is for data exchange between each pair of CAD, CAPP and CAM system. In fact, AP's for data exchange between CAD and CAPP, between CAPP and CAM, and between CAD and CAM is critical for CAD/CAPP/CAM integration. In these application protocols, the application reference models outline the requirements for data transfer, and the application interpreted models have common definitions of data for exchange. These common data models conduct the implementations of the exchange file processors and the data access interface.

Application protocols are also parts of STEP. Normative application protocols are essential for applications of STEP, because if different application protocols implemented on different systems for a particular application, these implementations would be incomparable. Each AP defines a data exchange standard for a defined family of products at a defined stage in its life cycle. Following are some of the common AP used,
AP 203 - pattern restricted 3D designs of mechanical parts and assemblies.

AP 210 - Electronic assembly interconnects and packaging design.

AP 214- Core data for automotive mechanical design processes

AP 224 - Mechanical product definition for process plans using machining features

AP 238 - STEP-NC Application interpreted model for computerized numerical controllers.

AP 239 - Product life cycle support

AP 240 - Process plans for machined products

\section{CONCLUSIONS}

STEP is the major effective method which gives a dominant sustains for /CAPP/ CAD/CNC \& CAM combination. The integrated assets of STEP provide a entire detail information of product data, for the global product information method of integration this method is the root for that. The technique for data interchange and distribution for integration completed by using the implementation methods of STEP. For exact applications, a variety of application rules are demanded for standard and steady implementations. The formal description of STEP assurances the possibility of software tools which support to the implementation methods, so as to the system integration.

\section{REFERENCES}

[1] Bloor, M. S. and Owen, J, "CAD/CAM product-data exchange: the next step”, CAD,23(1991),237.

[2] ISO CD10303-1, "STEP Part1: Overview and fundamental principles", ISO TC184/SC4/PMAG, 1992.

[3] Smith, B. M., "Product data exchange: the PDES project - status and objectives", NISTIR 89-4165, 1989.

[4] U.S. Product Data Association, "An American National Standard: Product Data Exchange using STEP (PDES) Part 41 - Integrated Generic Resources: Fundamental of Product Description and Support (ANS US PRO/IPO-200-041-1994," Trident Research Center, Suite 204,5300 International Blvd.,N. Charleston, SC.

[5] U.S. Product Data Association, "An American National Standard: Product Data Exchange using STEP (PDES) Part 203 - Application Protocol: Configuration Controlled Design (ANS US PRO/IPO-200-041-1994," Trident Research Center, Suite 204,5300 International Blvd.,N. Charleston, SC. 